\title{
Tendencia de la mortalidad por tumores cerebrales malignos en Chile. Análisis de tasas
}

\section{Analysis of Chilean trends in mortality \\ from malignant brain tumors}

Violeta Díaz T. ${ }^{1}$,Alfredo Yáñez L. ${ }^{2}$, Carolina Ponce $0 .{ }^{3}$, Rodrigo Villegas ${ }^{4}$ yJuan A. Pastén 5

\begin{abstract}
O bjective: To analyse the Chilean trends in mortality from brain malignancies between 1985 and 1999. Methods: We calculated mortality rates from malignant brain tumors using data obtained from death certificates available at the National Statistics Office. The following International Classification of Diseases categories were selected: 191.0 to 191.9 (ICD-9), and C71.0 to C71.9 (ICD-10). The rates were adjusted using direct standardization. Prais-Winsten methodology was used for time correlation analysis. Results: Sex-specific rates varied from 0.9 to 1.75 per 100.000 in men and from 0.7 to 1.22 in women. The trend was toward a statistically significant increase in mortality from malignant brain tumors in both groups. The analysis by age group showed no statistically significant variation in those below 35 years old, and a statistically significant increase in those between 35 and 39 years old, and in those above 45 years old. Conclusions: The trend in mortality from malignant brain tumors, in Chile, shows a statistically significant increase in those between 35 and 39 years old, and in those above 45 years old.
\end{abstract}

Key words: Trend of non metastatic malignant brain tumor mortality rates.

Rev Chil Neuro-Psiquiat 2006; 44(4): 263-270

Recibido: 14 mayo 2006

Aceptado: 2 noviembre 2006

1 Neuróloga, Epidemióloga Clínica.

2 Neurocirujano.

3 Bioquímica, Magíster en Ciencias.

4 Magíster en Bioestadística.

5 Magíster Epidemiología Clínica.

Hospital Clínico de la Universidad de Chile, Dr. José Joaquín Aguirre.

Unidad de Epidemiología Clínica, Universidad de Chile y Escuela de Salud Pública.

Sin conflictos de interés. 


\section{Introducción}

La mortalidad por tumores cerebrales representa el 2,4\% de las muertes por cáncer en EEUU; en niños es la segunda causa específica de muerte ${ }^{1}$. En Chile, la tasa de mortalidad por cáncer es de 119,4 por 100.000 representando el $23 \%$ de todas las muertes; la tasa de mortalidad por cáncer cerebral varía de 1 a 2 por 100.000 con proyección al ascenso ${ }^{2}$.

Los métodos diagnósticos para determinar los tumores cerebrales son de alto costo (tomografía cerebral y resonancia magnética cerebral) y no existe un método de tamizaje de laboratorio que facilite el proceso de diagnóstico; la mayor parte de los estudios corresponden a series de casos clínicos y no poblacionales. En los tumores cerebrales se llega al diagnóstico por su sintomatología y muy escasamente pueden aparecer como hallazgo incidental en exámenes hechos por otros propósitos diagnósticos. En las últimas décadas se ha descrito en varios países un aumento de la incidencia por tumores primarios del sistema nervioso central (SNC) especialmente debido al mejoramiento y la generalización en la detección de estos tumores con técnicas de imagen como la tomografía axial computarizada (TAC) y la resonancia magnética (RM $)^{3-6}$.

El objetivo del presente trabajo es evaluar la tendencia de la mortalidad por tumores primarios malignos en Chile en los últimos 15 años e identificar su distribución por edad y sexo.

\section{Material y Método}

Estudio descriptivo y correlacional hecho sobre el cálculo de tasas de mortalidad de datos obtenidos en los índices demográficos de Chile, entre los años 1985 a 1999. Se usaron las categorías 191,0 a 191,9 de la Clasificación Internacional de Enfermedades (CIE-9) y C71.0 a C71.9 de la CIE-10 (Tabla 1). Se utilizó la población de Chile de los años estudiados, ajustándose a la población de 1999. Se calcularon tasas específicas por edad y sexo utilizando método directo de ajuste de tasas. Se calculó la tendencia de la mortalidad por edad y sexo. Para su significado estadístico se utilizó la prueba de regresión de PraisWinster para mediciones correlacionales en el tiempo, usando el paquete estadístico Stata 7, obteniendo 95\% intervalo de confianza y considerando una probabilidad menor a 0,05 para su significado estadístico.

\section{Resultados}

Se recopilaron 2.304 TCM . Las tasas específicas por sexo varían entre 0, 90 (año 1986) y 1,74 (año 1996) por 100.000 en sexo masculino y 0,70 (año 1988) a 1,22 (año 1998) en las mujeres (tabla 2).

El estudio de los grupos etarios no demostró aumento significativo en aquellos de 44 o menos, excepto en el subgrupo de 35 a 39 años, en éste subgrupo se encontró un aumento estadísticamente significativo al igual que en todos los grupos etarios de mayores de 44. En grupos de 40 a 44 años las cifras comienzan a ascender a cifras de 0,28 (1989) a 1,94 (1995). Entre los grupos etarios de 45 años y más la tendencia es estadísticamente significativa para cada grupo etario. En los grupos mayores de 70 se encuentran cifras más altas llegando a valores de hasta 8,14 por 100.000 en 1996 (tabla 3, figuras 1, 2, 3 y 4).

El aumento en las tasas de mortalidad se da en ambos sexos, en mujeres es significativamente ascendente ( $R 2=0,68, p<0,005.95 \%$ IC 0,02-0,05), la tendencia en hombres es también significativamente ascendente ( $R 2=0,70, p=<0,005$, IC 0,04-0,08) (figura 1). Los hombres presentan Tasas más altas que las mujeres $(0,47, p<0,005$, IC $0,18-0,42$ )

\section{Comentario}

Las tasas de incidencia de los tumores cerebrales malignos y benignos primarios del SNC en EE.UU. son de alrededor de 11,4 casos por 100.000 persona-año (4,2 por 100.000 persona- 
año para benignos y 6,8 por 100.000 personaaño para malignos ${ }^{7}$. La cifra es más alta en hombres que en mujeres ( 11,8 versus 11,0 por 100.000 persona-año). En Chile, no existen trabajos sobre incidencia en tumores cerebrales y sólo un porcentaje muy bajo de ellos puede completar tratamiento con radioterapia y quimioterapia. La ausencia de centros especializados en neuropatología hace aún más inciertos los diagnósticos neuropatológicos de los pacientes en nuestro medio. Teniendo presente éstas limitaciones podríamos decir que las cifras encontradas en mortalidad de tumores malignos (1,5 por 100.000$)$ po- drían ser muy similares a las encontradas en incidencia

En EE.UU., se encontró un incremento de las tasas de mortalidad por tumores cerebrales primarios malignos desde 1973 a 0,7\% por año, explicado por el aumento también de la incidencia de $1,2 \%$ por año ${ }^{7}$. El aumento de las cifras de incidencia y mortalidad de los tumores malignos se da especialmente en los grupos de 65 años y más aumentando hasta 2,5\% por año desde $1980^{\circ}$. Este incremento en la mortalidad se debería al crecimiento exponencial en el número de TAC entre los años 1975 y 1977, asociándose a la

Tabla 1. Clasificación Internacional de Enfermedades CIE-9

\begin{tabular}{ll}
\hline $\begin{array}{l}\text { Clasificación } \\
\text { Internacional } \\
191\end{array}$ & Tumor maligno del encéfalo excluye espacio retroocular (190.1) y Nervios Craneales (192) \\
\hline 191.0 & Cerebro, excepto lóbulos y ventrículos \\
191.1 & Lóbulo frontal \\
191.2 & Lóbulo temporal \\
191.3 & Lóbulo parietal \\
191.4 & Lóbulo occipital \\
191.5 & Ventrículos \\
191.6 & Cerebelo \\
191.7 & Protuberancia (ambos) y bulbo raquideo \\
191.8 & Otros \\
191.9 & Partes no especificadas astroblastoma, astrocitoma, ependimoma, glioma, meduloblastoma, \\
& neuroepitelioma, oligodendroblastoma. \\
\hline & \multicolumn{1}{c}{ Clasificación Internacional deEnfermedades CIE-10 } \\
\hline Clasificación & Tumor maligno del encéfalo excluyenervios craneales (C72.2 - C72.3), tejido retrobulbar \\
Internacional & \\
\hline $\mathbf{1 1}$ & \\
\hline C71.0 & Tumor maligno del cerebro, excepto lóbulosy ventrículos, cuerpo calloso, supratentorial SAI \\
C71.1 & Tumor maligno del lóbulo frontal \\
C71.2 & Tumor maligno del lóbulo temporal \\
C71.3 & Tumor maligno del lóbulo parietal \\
C71.4 & Tumor maligno del lóbulo occipital \\
C71.5 & Tumor maligno del lóbulo ventrículo cerebralexcluye cuarto ventrículo \\
C71.6 & Tumor maligno decerebelo \\
C71.7 & Tumor maligno del pedúnculo cerebral cuarto ventrículo \\
C71.8 & Lesiones desitios contiguos del encéfalo \\
C71.9 & Tumor maligno del encéfalo, posiciones no especificadas \\
\hline
\end{tabular}


Tabla 2. Tasas ajustadas de mortalidad por tumores cerebrales malignos por $\mathbf{1 0 0 . 0 0 0}$ habitantes según sexo

\begin{tabular}{cccc}
\hline Años & Masculino & Femenino & Total \\
\hline 1985 & 0,95 & 0,77 & 0,86 \\
1986 & 0,90 & 0,77 & 0.84 \\
1987 & 0,92 & 0,74 & 0,83 \\
1988 & 0,73 & 0,70 & 0,71 \\
1989 & 0,91 & 0,82 & 0,86 \\
1990 & 1,09 & 1,09 & 1,09 \\
1991 & 1,59 & 0,78 & 1,18 \\
1992 & 1,27 & 0,90 & 1,08 \\
1993 & 1,38 & 1,04 & 1,21 \\
1994 & 1,47 & 1,16 & 1,31 \\
1995 & 1,55 & 1,17 & 1,36 \\
1996 & 1,74 & 1,22 & 1,48 \\
1997 & 1,46 & 0,96 & 1,21 \\
1998 & 1,59 & 1,22 & 1,40 \\
1999 & 1,49 & 1,20 & 1,35 \\
\hline
\end{tabular}

introducción de ésta nueva tecnología ${ }^{4,9}$. La introducción de la TAC y la RM serían responsables al menos en un $20 \%$ del incremento de los tumores de cerebro en conjunto con otros factores $^{10}$. La aparición del SIDA y su consiguiente aumento de los linfomas intracerebrales, puede contribuir al aumento de la incidencia de tumores cerebrales malignos, como también los cambios que han ocurrido en la clasificación de los tumores cerebrales primarios ${ }^{11}$. Éste punto aun no esta dilucidado. En Chile, la tomografía se introdujo masivamente durante la década del 90 , por lo que el factor de diagnóstico sería menos significativo.

Utilizando data de Connecticut Tumor Registry, Shoemberg encontró una tasa de incidencia en tumores cerebrales benignos y malignos de 5,9 por 100.000 entre 1955 y $1964^{12}$. Un estudio reciente de Karatsu, en Japón, encontró una incidencia de todos los tumores cerebrales de 10,97 por 100.000 (tasa ajustada por edad) siendo más prevalentes en las edades extremas de la vida, $5 \%$

Tabla 3. Tasas de mortalidad en los diferentes grupos etarios, ajustadas a población de 1999 por 100.000 habitantes

\begin{tabular}{|c|c|c|c|c|c|c|c|c|c|c|c|c|c|c|c|}
\hline ños & 85 & 86 & 887 & 988 & 989 & 990 & 1991 & 1992 & 1993 & 1994 & 995 & 1996 & 1997 & 1998 & 1999 \\
\hline & & & & & & & & & & & & & & & \\
\hline & & & & & & & & & & & & & & & 0,55 \\
\hline & ,78 & 3 & & & & & & & & & & & 57 & & 0,79 \\
\hline & 34 & & & & & & & & & & & & & & 0,32 \\
\hline &, 25 & 48 & 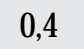 & 032 & & & 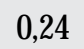 & & & & 0,65 & & 0,16 & & 0,17 \\
\hline & & & & & & & & & & & & & & & 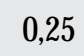 \\
\hline 3 & 79 & 2 & 0,6 & & & & & 0 & & & & & 0,66 & & 0,33 \\
\hline a3 & 0,75 & & & & & & & & & & & & 1,16 & & 1,1 \\
\hline 1 & 1,02 & 135 & 1,17 & 0,7 & 0,28 & 0,9 & 6 & 1, & 1 & 1,53 & 1,94 & & 1,48 & 2 & 1,28 \\
\hline a 4 & 0,36 & 1 & 1,6 & & & 1,4 & & & & & 1,92 & & 1,15 & & 1,56 \\
\hline 54 & 1,29 & 1,48 & 1,05 & 1,2 & 4 & 3 & & & & 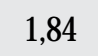 & 75 & & 3,5 & & 2,74 \\
\hline a 59 & 2,82 & 2,18 & 1,66 & 2,1 & 167 & & & & & 3,55 & 2,88 & & ,26 & & 4,35 \\
\hline & 1,5 & 3,93 & & & & & & & & & & & & & 4,64 \\
\hline a 69 & & 0,84 & 1,06 & 1,83 & 3,22 & & 6,77 & 4, & & 6,56 & 4,33 & 7 & 5,52 & 67 & 5,55 \\
\hline a 74 & & & & 2 & & & & & & & & & & & 6,49 \\
\hline a 79 & & & & 3,5 & $2,1,0$ & & 4,66 & & & & & & 5,85 & 7,77 & 8,57 \\
\hline 80 & & & & 3,0 & 2,9 & 2,8 & 2,74 & 3,33 & 4,55 & 5,63 & 6,66 & 8,14 & 5,03 & 7,00 & 2,6 \\
\hline
\end{tabular}




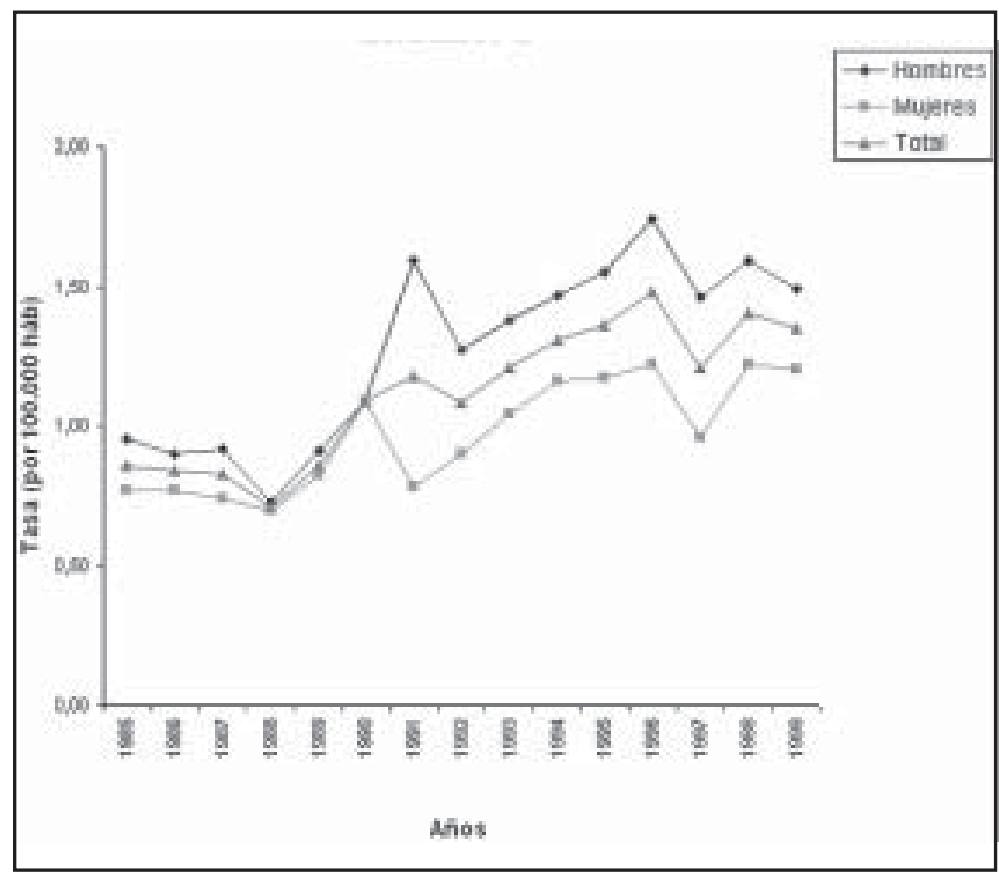

Figura 1. Seaprecia un aumento dela tendencia a la mortalidad por tumores malignos primarios del encéfalo tanto en hombres como en mujeres.

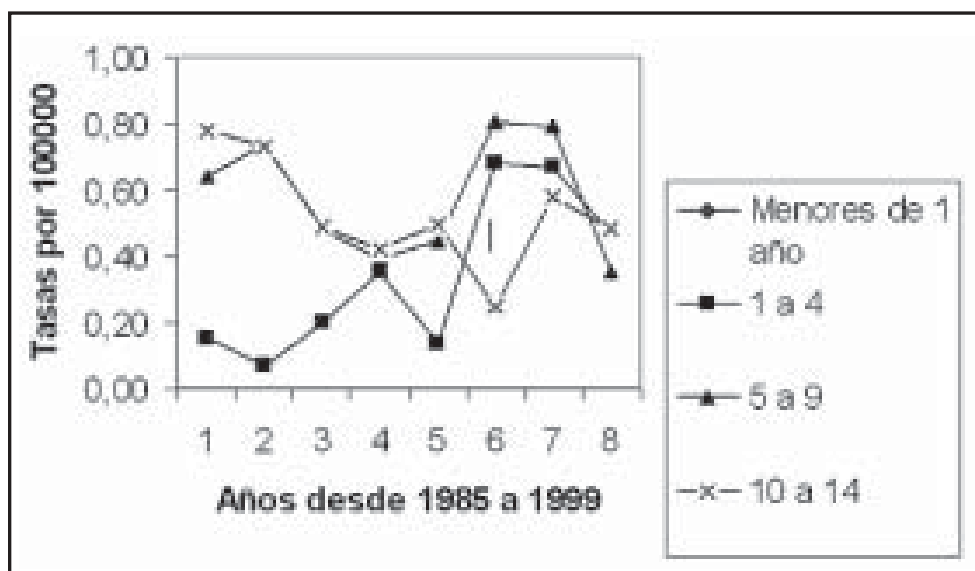

Figura 2. No existe un claro aumento de la mortalidad en los grupos menores de 14 años.

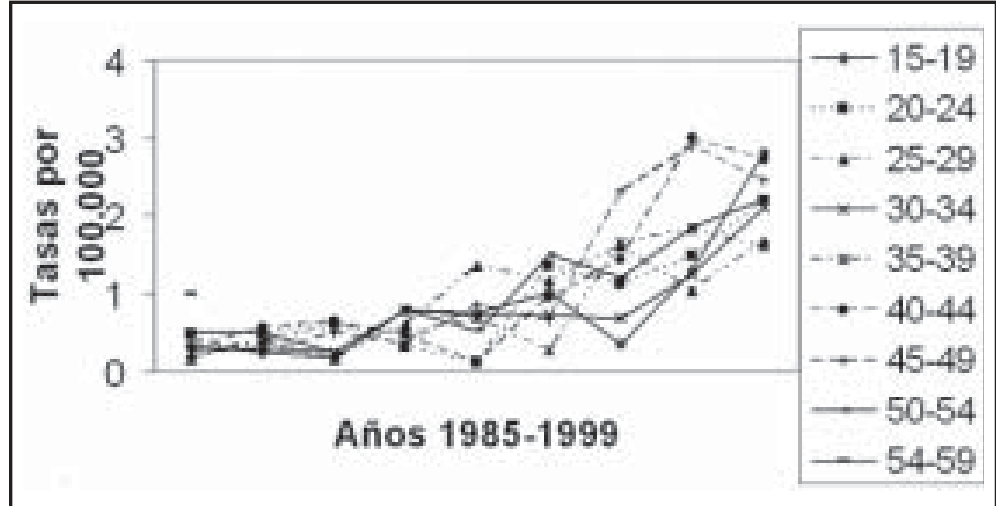

Figura 3. Claro aumento de la tendencia en la mortalidad sobre los 45 años. 


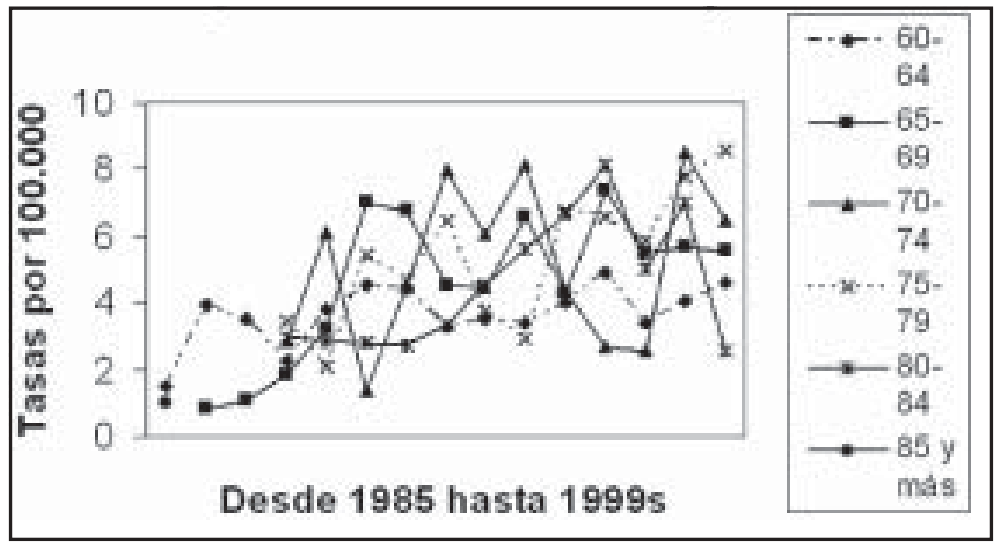

Figura 4. Existe una tendencia estadísticamente significativa sobre los 60 años. menores de 15 y 22,5\% mayores de 70 años 5 . En Inglaterra e Irlanda la incidencia de todos los tumores cerebrales es de 21 por 100.000 persona año $0^{13}$.

En el presente trabajo aparece una tendencia creciente a la mortalidad, en los grupos etarios de 35 a 39 años y sobre los 45 años, especialmente notorio en los mayores de 65 años, lo que concuerda con la literatura americana donde ocupa la cuarta causa específica de muerte por cáncer en hombres de 35 a 54 años ${ }^{1}$.

Es aún controvertida la estimación que existe una tendencia al aumento de la incidencia de tumores primarios cerebrales especialmente en niños y adultos mayores a través de todo el mundo en las últimas décadas, siendo la segunda causa tumoral en los niños después de la leucemia ${ }^{14}$. En el presente trabajo no encontramos aumento de la tendencia a la mortalidad en niños menores de 15 años y adultos menores de 35 años. La equivalencia de incidencia y mortalidad en niños es menos significativa en tumores malignos cerebrales, los niños tienen mejor pronóstico y el comportamiento biológico de los tumores es diferente.

La mayor parte de trabajos en tumores cerebrales se basan en estudios de series de casos y a través de ello se estiman incidencias y los estudios de incidencia se obtienen a través de datos de Registro de Cáncer; Kaatsch ${ }^{15}$ en Alemania entre los años 1990 a 1999 registró 3268 tumores cerebrales intracraneales e intraespinales en niños hasta los 15 años entregando una cifra de 2,6 por 100.000; comparable a las cifras de otros países que oscilan entre 1,7 a 4,1 por 100.000 , siendo los más frecuentes los astrocitomas $(41,7 \%)$, meduloblastoma $(18,1 \%)$ ependimoma $(10,4 \%)$ tumores neuroectodérmicos primarios $(6,7 \%)$ y craneofaringioma $(4,4 \%)$. La sobrevida a 5 años fue de $64 \%$.

En EE.UU., Ashan ${ }^{16}$ estudió tasas por edad y sexo para cada tipo específico de tumor cerebral y encontró un claro aumento de la incidencia, para todos los tipos de tumores con la edad. La tendencia comparada entre los períodos 19811982 y 1989 a 1990 también aumentaba 5,6 veces.

David FG y cols ${ }^{17}$ utilizando el Central Brain Tumor Registry en EE.UU., utilizando tasas específicas por edad y curvas de sobrevida entre 1985 a 1994 encontró incidencia de 13,8 por 100.000 y una prevalencia para el año 2000 de 130,8 por 100.000 para todos los tumores cerebrales. La sobrevida a 2 años fue de $58 \%$, a 5 años de $49 \%$ y a 10 años de $38 \%$, calculando que 350.000 individuos viven con el diagnóstico de tumor cerebral en el año 2000. La prevalencia para tumores malignos fue de 29,5 por 100.000 y de 97,5 por 100.000 para benignos

La creación de un registro de tumores cerebrales y la realización de conferencias y de consenso en definición y clasificación de tumores cerebrales, ha permitido una mejor vigilancia de los tumores cerebrales en el análisis de sobrevida, vigilancia epidemiológica y evaluación de terapéuticas en EE.UU. y otros países del mundo ${ }^{18,19}$. 


\section{Resumen}

Existe controversia en el aumento de la incidencia en las tasas de tumores primarios malignos de cerebro. Este incremento podría explicarse por el crecimiento exponencial en el número de Tomografías computarizadas. Objetivo: Evaluar la tendencia de la mortalidad por tumores cerebrales primarios malignos en Chile (TCM). Metodología: Estudio de tasas de mortalidad de datos obtenidos en índices demográficos (años 1985 a 1999). Se utilizó la población de Chile de los años estudiados y se ajustó a la población estimada de 1999. Se usó las categorías 191,0 a 191,9 y C 71-0 a C 71-9 de la Clasificación Internacional, correspondiendo al grupo de tumores malignos del SNC. Se estudiaron tasas específicas por edad y sexo. Se calculó la tendencia utilizando las tasas ajustada por edad y sexo. Se utilizó una prueba de regresión lineal (Prais-Winsten) para mediciones correlacionales en el tiempo (Stata 7). Resultados: Se obtuvo 2.304 TCM . Las tasas específicas por sexo varían entre 0,90 a 1,75 x 100.000 en hombres y de 0,7 a 1,22 en mujeres. La tendencia global de la mortalidad por tumores tiende al ascenso en ambos grupos y es significativamente más alta en hombres $(0,4795 \%$ IC 0,18 a 0,42 p = <0,005) El estudio por grupos etarios no muestra un aumento significativo en menores de 14 años, ni entre 15 y 34 años. En los grupos de 45 años y más es estadísticamente significativa. Conclusión: La tendencia a la mortalidad por TCM aparece en ascenso en los grupos etarios 35 a 39 años y 45 años y más

Palabras clave: Tumores malignos cerebrales y mortalidad.

\section{Referencias}

1. Central Brain Tumor Registry of United States. 1995 Annual Report 1996. Cancer Statistics 1996. CA: A Cancer Journal for Clinicians 1996; 46: 8-9, 19.

2. Tasas de mortalidad ajustadas por edad 1985 1999 y proyecciones de mortalidad 2000-2010 según causa específica y sexo. Chile 1985-2010. Instituto Nacional de Estadísticas de Chiley M inisterio de Salud Pública. Anuario 2002.

3. Davis F G, Kupelian V, Freels S, MacCarthy B. Prevalence estimate for primary brain tumors in USA by behavior and mayor histology groups. Neuro-Oncology 2001; 3: 152-8.

4. Greig N H, Ries L G, Yancik R, Papoport S I. Increasing annual incidence of primary malignant brain tumors in the elderly. J Natl Cancer Inst 1990; 82: 1621-4.

5. Karatsu J, Takeshima H, Ushio Y. Trends in the incidence of primary intracraneal tumors in Kumamoto, Japan. Int J Clin Oncol 2001, 6: 18391.
6. Jukich P J, M acCarthy B J K, Surawicz T S, Freels S, Davis $F$ G. Trend in incidence of primary brain tumor in the United States 1985-1994. NeuroOncology 2001; 3: 141-51.

7. Ries $L A G$, M iller B A, Hankey B F. SEER cancer statistics review 1973-1991: Tables and Graphs, Bethesda, Md: Demographic Analysis Section, Biometry Branch, Division of Cancer Prevention and Control, National Cancer and Control, National Cancer Institute; 1994. NIH Publication 94-2789.

8. Modan B, Wagener D K, Feldman J J. Increased mortality from brain tumor: A combined outcome of diagnostic technology and change of attitude toward the elderly. Am J Epidemiology 1992; 135: 1349-57.

9. Desmeules M, Mikkelsen T, Mao Y. Increasing incidence of primary malignant brain tumors: Influence of diagnostic methods. J Natl Cancer Inst 1992; 84: 442-5.

10. Riggs J $E$, Ketonen $L M$. The initial impact of 
computed tomography on mortality attributed to brain tumor. Neuroimaging 1992; 2 (3): 136-8.

11. Davis F G, M acCarthy. Epidemiology of Brain Tumor. Current opinion in Neurology 2000, 13: 63540.

12. Schoemberg B S, Christine B, Whisnant J P. The descriptive epidemioloy of primary intracraneal neoplasm. Arch Neurol 1981; 38: 217.

13. Ogungho B I, Najim O, M endelow A D, Crawford $P$ J. Epidemiology of adult brain tumor in Great Britain and Ireland. Brt J Neurosurgery 2002; 16: 140-5.

14. Nomura S, Nishizaki T, Yamashita K. Pediatric Brain tumors in 10 years periods from 1986 to 1995 in Yamaguchi prefecture: epidemiology and comparison with adult brain tumors. Pediatric neurosurgery 1998; 28: 130-4.

15. Kaatsch P, Rickert $\mathrm{CH}$, Kuhl J. Population-based epidemiologic data on brain tumor in German Children. Cancer 2001; 15: 3155-64.

16. Ashan H, Neugut Al, BruceJ N. Trends in incidence of primary malignant brain tumors in USA 19811990. Intern J Epidemiology 1995, 24: 1078-85.

17. David F G, M acCarthy B J, Berger M S. Centralized database avaible for describing primary brain tumor incidence, survival, and treatment: Central Brain Tumor Registry of the United States; Surveillance, Epidemiology and Ends results and National Center Database. Neuro-oncology 1999; 1: 205-11.

18. MacCarthy B, Surawicz T, Bruner J, et al. Consensus Conference on Brain Tumor definition for registration. Neuro-Oncology 2002; 4: 1-99.

19. Davis F G, Kupelian V, Freels S. Prevalence estimates for primary brain tumor in the Unites States. Neuo-Oncology 2001; 3: 152-8.

Correspondencia:

Violeta Díaz T.

Servicio de N eurología y N eurocirugía,

Hospital Clínico de la Universidad de Chile.

Santos Dumont 999, Santiago de Chile.

E-mail:vidiaz@med.uchile.cl 